\title{
Changes in brain function during negative emotion processing in the long-term course of depression
}

Verena Enneking*, Melissa Klug*, Tiana Borgers, Katharina Dohm, Dominik Grotegerd,

Lisa Marie Frankenberger, Carina Hülsmann, Hannah Lemke, Susanne Meinert, Elisabeth J. Leehr, Nils Opel, Janik Goltermann, Maike Richter, Lena Waltemate, Joscha Böhnlein, Lisa Sindermann, Jonathan Repple, Jochen Bauer, Mareike Thomas, Udo Dannlowski* and Ronny Redlich*

\section{Background}

Relapses in major depression are frequent and are associated with a high burden of disease. Although short-term studies suggest a normalisation of depression-associated brain functional alterations directly after treatment, long-term investigations are sparse.

\section{Aims}

To examine brain function during negative emotion processing in association with course of illness over a 2-year span.

\section{Method}

In this prospective case-control study, 72 in-patients with current depression and 42 healthy controls were investigated during a negative emotional face processing paradigm, at baseline and after 2 years. According to their course of illness during the study interval, patients were divided into subgroups ( $n=25$ no-relapse, $n=47$ relapse). The differential changes in brain activity were investigated by a group $\times$ time analysis of covariance for the amygdala, hippocampus, insula and at whole-brain level.

\section{Results}

A significant relapse $\times$ time interaction emerged within the amygdala $\left(P_{\text {TFCE-FWE }}=0.011\right)$, insula $\left(P_{\text {TFCE-FWE }}=0.001\right)$ and at the whole-brain level mainly in the temporal and prefrontal cortex $\left(P_{\text {TFCE-FWE }}=0.027\right)$, resulting from activity increases within the no-relapse group, whereas in the relapse group, activity decreased during the study interval. At baseline, the no-relapse group showed amygdala, hippocampus and insula hypoactivity compared with healthy controls and the relapse group.

\section{Conclusions}

This study reveals course of illness-associated activity changes in emotion processing areas. Patients in full remission show a normalisation of their baseline hypo-responsiveness to the activation level of healthy controls after 2 years. Brain function during emotion processing could further serve as a potential predictive marker for future relapse.

\section{Keywords}

Depressive disorders; imaging; relapse; emotion processing; limbic system.

\section{Copyright and usage}

(C) The Author(s), 2022. Published by Cambridge University Press on behalf of the Royal College of Psychiatrists. This is an Open Access article, distributed under the terms of the creative commons Attribution licence (https://creativecommons.org/ licenses/by/4.0/), which permits unrestricted re-use, distribution, and reproduction in any medium, provided the original work is properly cited.
Major depressive disorder is one of the most common and burdening mental diseases worldwide, ${ }^{1}$ and is associated with a mood-congruent bias in emotion processing. ${ }^{2,3}$ The course of the illness shows a large interpersonal heterogeneity, varying from one single lifetime depressive episode to recurrent or ongoing episodes, with the risk for a future relapse being increased with each episode. ${ }^{1,4}$ In fact, it is the course of illness over years that determines chronic disease burden and loss of functioning. ${ }^{5}$ The neural correlates that underlie depressive relapses are of high psychosocial and economic interest, albeit poorly understood. Prospective neuroimaging studies could help to understand how disease progression affects the brain function during emotion processing, and identify patients at high risk of relapse who could benefit from applying intensified treatments and prevention strategies.

\section{Neural correlates of negative emotion processing in depression}

On the neural level, the mood-congruent bias in emotion processing in depression has been linked to a dysfunction of a fronto-limbic network, with a hyperactivity of (para-)limbic regions - such as the amygdala, hippocampus and insula - and a hypoactivity of prefrontal regions, including the dorsomedial and dorsolateral prefrontal cortex (DLPFC), in response to negative stimuli. ${ }^{2,3}$ So far,

* V.E. and M.K. are joint first authors; U.D. and R.R. are joint senior authors. longitudinal functional magnetic resonance imaging (fMRI) studies mainly focused on short-term effects of treatments for depression on brain function, mostly indicating a normalisation of depression-associated alterations in brain activity after successful treatment; for example, a reduction of pre-treatment amygdala and hippocampus hyperactivity during processing of sad or fearful faces was reported after treatment with antidepressants ${ }^{6}$ and electroconvulsive therapy. ${ }^{7}$ Regarding the insula, the state of research is less clear, with some studies indicating activity increases to negative stimuli after treatment, ${ }^{6}$ and other studies pointing to decreases. ${ }^{6,8}$

\section{Long-term fMRI investigations}

Only two fMRI studies examined changes in brain function in patients with depression during emotion processing covering more than 1 year. ${ }^{9,10}$ They revealed that in remitted patients, insula activity in response to positive stimuli was reduced after 2 years, ${ }^{10}$ and symptomatic improvement was associated with increased amygdala and hippocampus activity in response to positive and negative words. ${ }^{9}$ However, regarding negative stimuli, these results seem to stand in contrast to evidence of short-term treatment studies described above, which mainly showed a reduction in limbic hyperactivity. ${ }^{6,8}$ As Opmeer et $\mathrm{al}^{10}$ found no activity changes for negative stimuli, there is a need to further investigate the long-term changes in brain function during negative stimuli processing.

Furthermore, the study of $\mathrm{Ai}$ et $\mathrm{al}^{9}$ suggested that brain functional changes were a result of remission status rather than 
of duration of depression during the study interval, whereas Opmeer et $\mathrm{al}^{10}$ did not examine the influence of duration in depression or relapses during the study interval on their results. Therefore, in depression, the impact of course of illness and remission status on brain functional changes is still unclear and remains to be explored.

\section{Aims of the study}

The present study was designed to examine course of illnessdependent changes in negative emotion processing in a prospective approach. As the few longitudinal studies that examined brain functional changes over the long-term course of depression pointed to changes in the amygdala, hippocampus and insula as parts of brain circuits relevant for emotion processing and salience detection, a particular focus was placed on these regions. The objectives were as follows: (a) to investigate long-term changes in brain function during negative emotion processing and its dependence on the course of illness and remission status in patients with depression, in a prospective naturalistic design; (b) to subsequently control for the influence of medication dose and psychotherapy on brain function; and (c) to identify baseline differences in brain activity between patients with depression with different course of illness (full remission versus recurrent or ongoing depression).

\section{Method}

\section{Participants and study design}

In this case-control study, participants were investigated as part of an ongoing study of the Münster Neuroimaging Cohort from May 2010 to June 2015, and were reassessed from September 2012 to May 2018 (interscan interval: mean 2.25 years, s.d. 0.30 years, range 1.92-3.33 years). At baseline, all patients were experiencing a moderate-to-severe depressive episode and were receiving inpatient treatment (within the Department of Psychiatry at University Hospital of Münster or within the Psychiatric Hospital of the Landschaftsverband Westfalen-Lippe in Münster). Healthy controls were recruited by newspaper announcements and public notices. Participants received a financial compensation. The authors assert that all procedures contributing to this work comply with the ethical standards of the relevant national and institutional committees on human experimentation and with the Helsinki Declaration of 1975, as revised in 2008. All procedures were approved by the ethics committee of the University of Münster (2007-307-f-S). Written informed consent was obtained from all participants.

Exclusion criteria for all participants were any neurological abnormalities, chronic medical diseases, a history of alcohol or substance dependence, electroconvulsive therapy, benzodiazepine intake at study time, or any magnetic resonance imaging contraindications. For patients with depression, a further exclusion criterion was a diagnosis of bipolar or psychotic disorder, whereas healthy controls had to be free from any lifetime psychiatric disorder at baseline and follow-up (for a visualisation of exclusion process, see Supplementary Fig. 1 available at https://doi.org/10.1192/bjp. 2021.223).

At baseline and follow-up, participants underwent fMRI during processing of negative emotional faces. Furthermore, the structured clinical interview for the DSM-IV ${ }^{11}$ and the Hamilton Rating Scale for Depression (HRSD) ${ }^{12}$ were conducted by trained clinical raters. Course of illness was assessed at baseline and follow-up. Type and frequency of psychotherapeutic interventions before baseline and during the study interval were documented. To quantify psychopharmacological treatment at baseline and follow-up, a composite medication load index was computed for each time point (see
Supplementary Methods 1.1). For details on psychopharmacological medication intake, see Supplementary Table 1.

The final sample comprised 72 patients with depression and 42 healthy controls. Participants' characteristics are summarised in Table 1 . There was an overlap of 53 patients with depression and 31 healthy controls with a sample reported in one of our earlier studies investigating long-term morphological brain changes. ${ }^{13}$ The patient group was further divided into two subgroups following a previously published protocol: ${ }^{13,14}$ patients who were in full remission at follow-up and who did not experience any further depressive episode after baseline (no-relapse group, $n=25$ ), and patients who suffered from ongoing depression since baseline or experienced at least one further depressive episode according to the DSM-IV ${ }^{15}$ after baseline (relapse group, $n=47$ ).

\section{fMRI methods}

fMRI data acquisition and preprocessing followed previously published protocols. ${ }^{16,17}$ For details regarding the fMRI paradigm, data acquisition and preprocessing methods, see Supplementary Methods $1.2-1.4$ and previous works. ${ }^{16-18}$ Briefly, $\mathrm{T}^{*}$ functional data were acquired with a single-shot echoplanar sequence with a 3T scanner (Gyroscan Intera 3T, Philips Medical System, Best, The Netherlands). The fMRI paradigm was a modified version of the face-matching task described by Hariri et al. ${ }^{18}$ It consisted of four blocks of an emotional face processing task and five blocks of a sensorimotor control task. Faces were derived from the Ekman and Friesen stimulus set ${ }^{19}$ and expressed either anger or fear (Supplementary Fig. 2(a)), whereas the control task consisted of geometric shapes (circles or ellipses, see Supplementary Fig. 2(b)).

Onsets and durations of the two experimental conditions (faces and shapes) were modelled with a canonical hemodynamic response function in the context of the general linear model. The model was corrected for serial correlations and a high-pass filter of $128 \mathrm{~s}$ was applied to remove low-frequency noise. The six movement parameters from the realignment step were further entered as nuisance covariates. For each participant and measurement, an individual contrast image (faces $>$ shapes) was generated in each fixed-effects first-level analysis, which was then used in the following second-level, random-effects group analyses. For quality control, effects of interest were visually inspected for each participant and time point.

\section{Statistical analyses}

Analyses of demographic and clinical data were performed with SPSS Statistics (version 25.0 for Windows; IBM Corporation). Second-level analyses of fMRI data were conducted with statistical parametric mapping software (SPM12, version 6685 for MATLAB on Ubuntu; Wellcome Department of Cognitive Neurology, London, UK; http://www.fil.ion.ucl.ac.uk/spm). Significance thresholds for multiple testing were obtained at the cluster-level by threshold-free cluster enhancement (TFCE), using the TFCE toolbox (version 223; Structural Brain Mapping Group, Jena, Germany; http://dbm.neuro.uni-jena.de/tfce) implemented in SPM12. We established a family-wise error (FWE)-corrected threshold of $P<0.05$, obtained by 10000 permutations per test. All second-level analyses were performed based on the faces $>$ shapes contrast.

\section{Region-of-interest analyses}

First, all second-level analyses were performed in a region-of-interest (ROI) approach, based on masked voxel-wise analyses using small volume correction as implemented in the TFCE toolbox in 


\begin{tabular}{|c|c|c|c|c|c|}
\hline & $\begin{array}{l}\text { Patients with depression } \\
\text { and relapse, } n=47\end{array}$ & $\begin{array}{l}\text { Patients with depression } \\
\text { and no-relapse, } n=25\end{array}$ & $\begin{array}{c}\text { Healthy } \\
\text { controls, } \\
n=42\end{array}$ & & \\
\hline Variable & Mean (s.d.) & Mean (s.d.) & Mean (s.d.) & $P$-value ${ }^{a}$ & $P$-value ${ }^{b}$ \\
\hline Interscan interval, years & $2.20(0.31)$ & $2.21(0.21)$ & $2.34(0.31)$ & 0.07 & 0.91 \\
\hline \multicolumn{6}{|l|}{ Sociodemographic characteristics } \\
\hline Age & $37.5(12.4)$ & $32.8(10.6)$ & $37.4(9.1)$ & 0.17 & 0.11 \\
\hline Gender (female/male), number of patients & $27 / 20$ & 13/12 & 19/23 & $0.52^{\mathrm{c}}$ & $0.66^{\mathrm{c}}$ \\
\hline \multicolumn{6}{|l|}{ Symptom severity } \\
\hline HRSD baseline & $23.2(4.9)$ & $22.6(3.6)$ & $0.5(0.8)$ & $<0.001$ & 0.59 \\
\hline HRSD follow-up & $12.7(7.7)$ & $4.0(4.0)$ & $1.1(1.9)$ & $<0.001$ & $<0.001$ \\
\hline \multicolumn{6}{|l|}{ Clinical characteristics at baseline } \\
\hline Number of depressive episodes before baseline & $4.9(6.6)$ & $3.4(4.7)$ & - & - & 0.33 \\
\hline Number of in-patient treatments before baseline & $2.2(1.7)$ & $1.4(0.9)$ & - & - & 0.009 \\
\hline Duration of in-patient treatment before baseline, months & $2.6(3.5)$ & $1.5(2.4)$ & - & - & 0.17 \\
\hline $\begin{array}{l}\text { Cumulative duration of depression before baseline, } \\
\text { months }\end{array}$ & $36.2(37.2)$ & $12.7(12.3)$ & - & - & $<0.001$ \\
\hline Acute comorbidity (yes/no), number of patients & $19 / 28$ & $6 / 19$ & - & - & $0.16^{\mathrm{C}}$ \\
\hline $\begin{array}{l}\text { Acute comorbid anxiety disorder (yes/no), number of } \\
\text { patients }\end{array}$ & $13 / 34$ & $5 / 20$ & - & - & $0.48^{\mathrm{C}}$ \\
\hline \multicolumn{6}{|l|}{ Clinical characteristics at follow-up } \\
\hline $\begin{array}{l}\text { Number of depressive episodes between baseline and } \\
\text { follow-up }\end{array}$ & $1.4(0.8)$ & $0.0(0.0)$ & - & - & $<0.001$ \\
\hline $\begin{array}{l}\text { Number of in-patient treatments between baseline and } \\
\text { follow-up }\end{array}$ & $0.6(0.7)$ & $0.0(0.0)$ & - & - & $<0.001$ \\
\hline $\begin{array}{l}\text { Remission status at follow-up (no remission/partial } \\
\text { remission/full remission), number of patients }\end{array}$ & $14 / 19 / 14$ & $0 / 0 / 25$ & - & - & $<0.001^{\mathrm{c}}$ \\
\hline Acute comorbidity (yes/no), number of patients & $22 / 25$ & $2 / 23$ & - & - & $<0.001^{\mathrm{c}}$ \\
\hline $\begin{array}{l}\text { Acute comorbid anxiety disorder (yes/no), number of } \\
\text { patients }\end{array}$ & $16 / 31$ & $2 / 23$ & - & - & $0.02^{c}$ \\
\hline \multicolumn{6}{|l|}{ Medical treatment } \\
\hline $\begin{array}{l}\text { Number of patients under psychopharmacological } \\
\text { medication at baseline (yes/no) }\end{array}$ & $44 / 3$ & $24 / 1$ & - & - & $0.67^{\mathrm{c}}$ \\
\hline $\begin{array}{l}\text { Number of patients under psychopharmacological } \\
\text { medication at follow-up (yes/no) }\end{array}$ & $38 / 9$ & $13 / 12$ & - & - & $0.01^{\mathrm{c}}$ \\
\hline Medication load index at baseline & $2.2(1.4)$ & $1.9(0.9)$ & - & - & 0.34 \\
\hline Medication load index at follow-up & $1.7(1.4)$ & $0.9(1.1)$ & - & - & 0.02 \\
\hline \multicolumn{6}{|l|}{ Psychotherapeutic treatment } \\
\hline $\begin{array}{l}\text { Number of psychotherapeutic treatments before } \\
\text { baseline (no information/0/1/ } \geq 2 \text { ), number of patients }\end{array}$ & 19/13/11/4 & $11 / 8 / 6 / 0$ & - & - & $0.33^{\mathrm{C}}$ \\
\hline $\begin{array}{l}\text { Number of patients under psychotherapeutic treatment } \\
\text { during study interval }^{\mathrm{d}} \text { (yes/no) }\end{array}$ & $35 / 12$ & $14 / 11$ & - & - & $0.88^{\mathrm{C}}$ \\
\hline Cognitive-behavioural therapy & 14 & 10 & - & - & - \\
\hline Psychoanalytic psychotherapy & 1 & 0 & - & - & - \\
\hline Psychodynamic psychotherapy & 7 & 0 & - & - & - \\
\hline Other method & 2 & 0 & - & - & - \\
\hline No information regarding method & 11 & 4 & - & - & - \\
\hline
\end{tabular}

SPM. The three a priori defined ROIs were the bilateral amygdala, bilateral hippocampus with parahippocampal gyrus and bilateral insula, each created separately by means of the Wake Forest University PickAtlas ${ }^{20}$ tool (version 3.0; NeuroImaging Tools \& Resources Collaboratory; https://www.nitrc.org/projects/wfu_pickatlas) implemented in SPM12 according to the automated anatomical labeling atlas ${ }^{21}$ definitions. Subregions were labelled by using cytoarchitectonic probability maps, implemented in the JuBrain Anatomy Toolbox ${ }^{37}$ (version 2.2b; Forschungszentrum Jülich, Jülich, Germany; https://www.fz-juelich.de/inm/inm-7/DE/Resources/ _doc/SPM\%20Anatomy\%20Toolbox_node.html) implemented in SPM12. A $3 \times 2$ analysis of covariance (ANCOVA) was computed with group (no-relapse, relapse, healthy controls) as between-participants factor, time (baseline, 2-year follow-up) as a within-participant factor, and age and gender as covariates. We investigated the relapse (no-relapse, relapse) $\times$ time (baseline, follow-up) interaction, the main effects of time and main effects of group at baseline and follow-up.

\section{Whole-brain analyses}

All second-level analyses for the $3 \times 2$ ANCOVA described for the ROI analyses were also performed at the whole-brain level.

\section{Additional analyses}

The Supplementary Methods provide a description of our additional analyses on (a) effects of medication dose and psychotherapy on changes in brain function (Supplementary Methods 1.5.1), (b) relapse prediction based on baseline brain functional data compared with clinical data (Supplementary Methods 1.5.2) and (c) effects of remission status on follow-up brain activity (Supplementary Methods 1.5.3). 
(a)

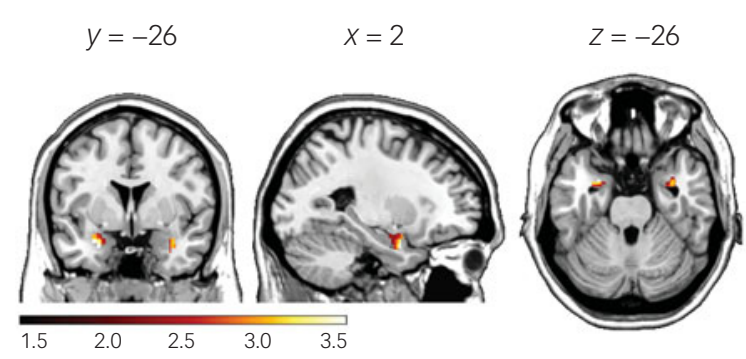

(c)

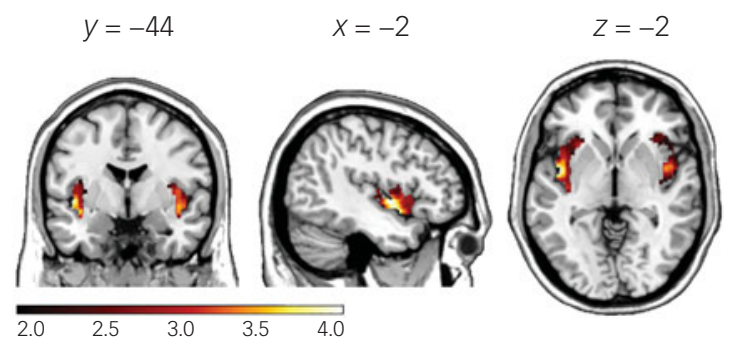

(b)

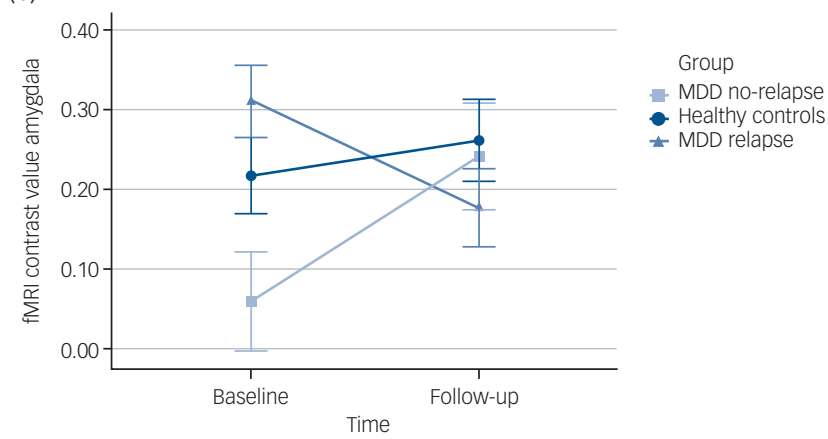

(d)

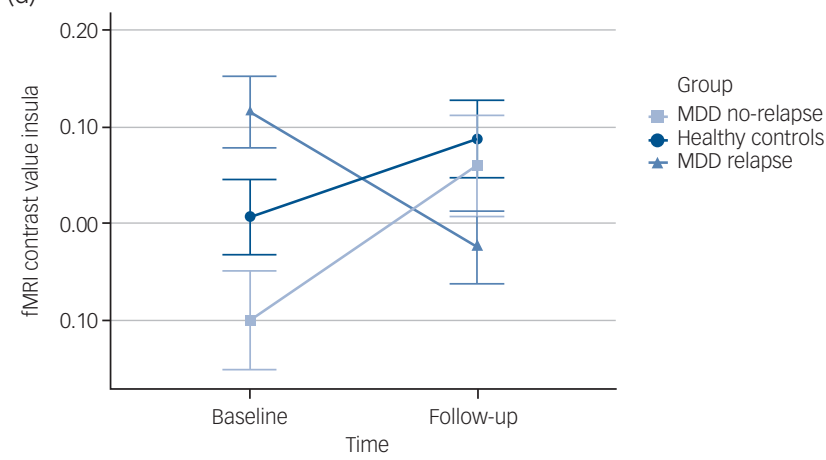

Fig. 1 Longitudinal relapsextime interaction (one-tailed) of amygdala and insula ROI analyses. (a) Significant clusters (left: $x=-26, y=2, z$ $=-26, t_{(220)}=3.32, k=104, P_{\mathrm{TFCE}-\mathrm{FWE}}=0.011$; right: $\left.x=34, y=0, z=-20, t_{(220)}=3.08, k=81, P_{\mathrm{TFCE}-F W E}=0.017\right)$ of the amygdala ROI analysis for the relapsextime interaction effect (one-tailed), driven by activity increases in the no-relapse group and activity decreases in the relapse group. The figure displays clusters significant at $P_{\mathrm{TFCE}-\mathrm{FWE}}<0.05$. Bar indicates $t$-values. (b) Plot depicts typical amygdala responses at baseline and follow-up for healthy controls, and patients with MDD with relapse and no-relapse during the study interval. fMRI contrast values were computed by extracting the first Eigenvariate of the significant left cluster $\left(x=-26, y=2, z=-26, t_{(220)}=3.32, k=104, P_{\mathrm{TFCE}-\mathrm{FWE}}=0.011\right)$ resulting from the amygdala ROI analysis of the relapsextime interaction (one-tailed). Error bars indicate 1 s.e.m. (c) Significant clusters (left: $x=-44, y=-2, z=-2$, $t_{(220)}=4.18, k=1350, P_{\text {TFCE-FWE }}=0.001$; right: $\left.x=44, y=2, z=-8, t_{(220)}=3.81, k=1088, P_{\text {TFCE-FWE }}=0.004\right)$ of the insula ROI analysis for the relapsextime interaction effect (one-tailed), driven by activity increases in the no-relapse group and activity decreases in the relapse group. The figure displays clusters significant at $P_{\text {TFCE-FWE }}<0.05$. Bar indicates $t$-values. (d) Plot depicts typical insula responses at baseline and follow-up for healthy controls, and patients with MDD with relapse and no-relapse during the study interval. fMRI contrast values were computed by extracting the first Eigenvariate of the significant left cluster $\left(x=-44, y=-2, z=-2, t_{(220)}=4.18, k=1350, P_{\text {TFCE-FWE }}=0.001\right)$ resulting from the insula ROI analysis of the relapsextime interaction (one-tailed). Error bars indicate 1 s.e.m. fMRI, functional magnetic resonance imaging; FWE, family-wise error; MDD, major depressive disorder; ROI, region of interest; TFCE, threshold-free cluster enhancement.

\section{Results}

\section{ROI analyses}

Longitudinal relapsextime interaction

There was a significant relapsextime interaction (one-tailed) for the bilateral amygdala (Fig. 1(a) and (b)) and bilateral insula (Fig. 1(c) and (d)). The post hoc $t$-tests for changes in brain function from baseline to follow-up within groups revealed that the relapsextime interaction resulted from significant amygdala and insula activity increases within the no-relapse group, whereas within the relapse group, insula activity was reduced from baseline to follow-up. Healthy controls showed no significant changes over time (see also Supplementary Table 2).

For the hippocampus ROI, no significant relapsextime interaction effect emerged $\left(P_{\text {TFCE-FWE }}=0.055\right)$. There was no significant main effect of time for any of the three ROIs (all $P_{\text {TFCE-FWE }}>0.126$ ).

\section{Cross-sectional group differences at baseline}

Supplementary Table 3 summarises the results of the betweengroup differences in baseline activity for all three ROIs. Briefly, a main effect of group at baseline emerged for the right amygdala, bilateral hippocampus and bilateral insula. Post hoc t-tests revealed that the no-relapse group showed hypoactivity within the left amygdala, bilateral hippocampus and bilateral insula, compared with healthy controls. Furthermore, patient groups differed in baseline activity, with higher baseline activity in the relapse group in the bilateral amygdala (Supplementary Fig. 3(a)), bilateral hippocampus (Supplementary Fig. 3(b)) and bilateral insula (Supplementary Fig. 3(c)), compared with the no-relapse group. Comparing the complete patient group with depression (norelapse and relapse groups together) with healthy controls, no difference in brain function emerged in any of the three ROIs (all $\left.P_{\text {TFCE-FWE }}>0.150\right)$.

\section{Cross-sectional group differences at follow-up}

There was no main effect of group at follow-up for any of the three ROIs (all $P_{\text {TFCE-FWE }}>0.187$ ).

\section{Whole-brain analyses}

\section{Longitudinal relapsextime interaction}

Figure 2(a) and (b) and Supplementary Table 4 display the results of the relapsextime interaction (one-tailed) at the whole-brain level. Briefly, a significant relapsextime interaction emerged, driven by 
(a)

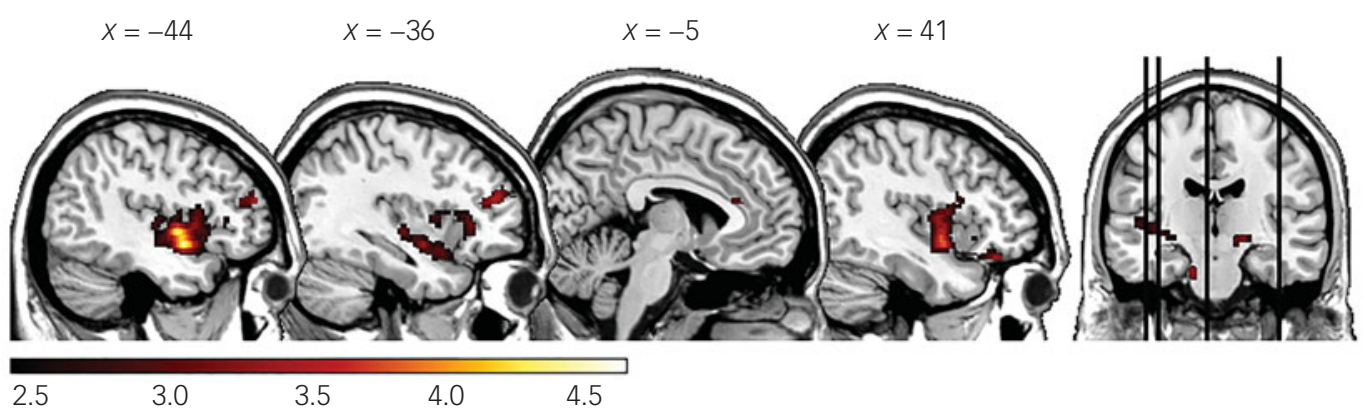

(b)
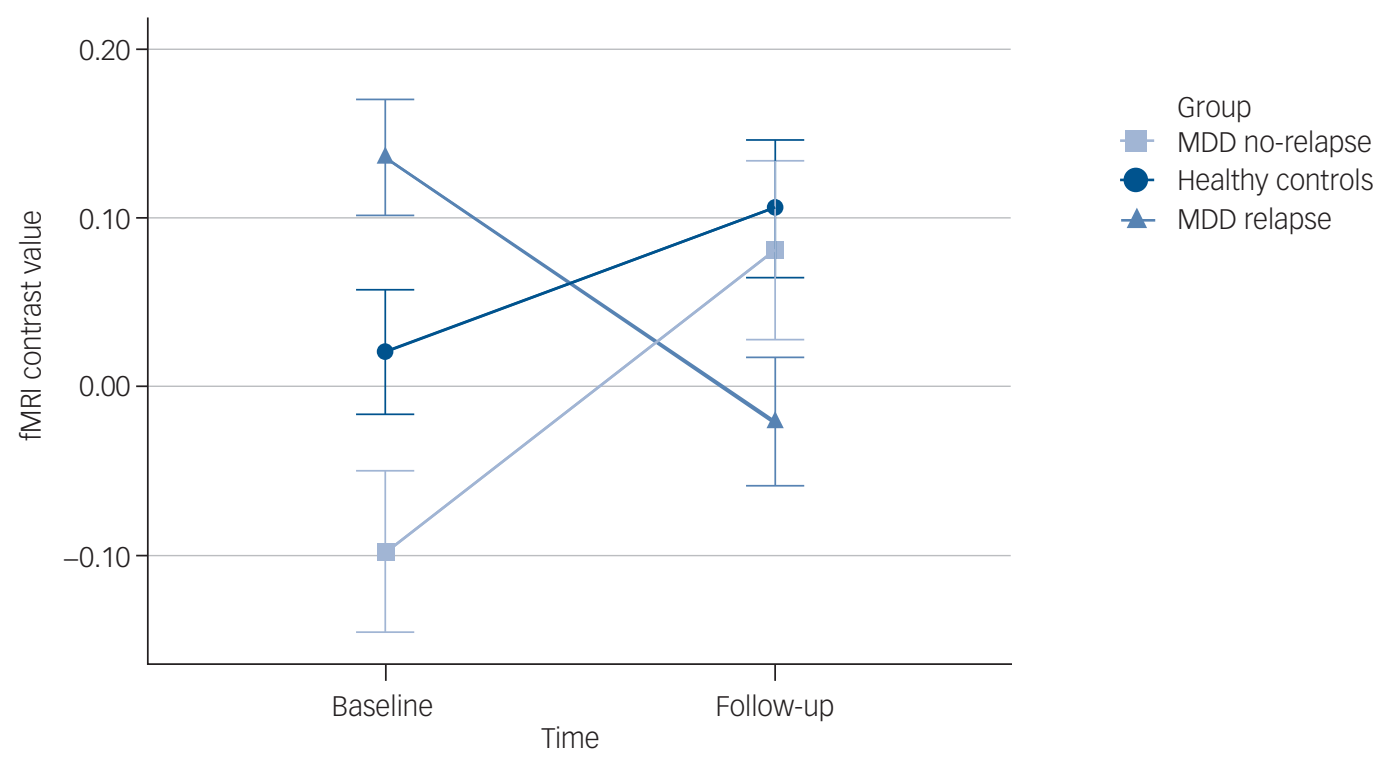

Fig. 2 Longitudinal relapsextime interaction (one-tailed) of whole-brain analysis. (a) Significant clusters of the whole-brain analysis for the relapsextime interaction effect (one-tailed), driven by activity increases in the no-relapse group and activity decreases in the relapse group. The figure displays clusters significant at $P_{\mathrm{TFCE}-\mathrm{FWE}}<0.05$. Bar indicates $t$-values. (b) Plot depicts typical responses at baseline and follow-up for healthy controls, and patients with MDD with relapse and no-relapse during the study interval. fMRI contrast values were computed by extracting the first Eigenvariate of the largest significant cluster $\left(x=-44, y=2, z=-10, t_{(220)}=4.32, k=2190, P_{\text {TFCE-FWE }}=0.027\right)$ resulting from the whole-brain analysis of the relapsextime interaction (one-tailed). The cluster included parts of the following regions: insula, superior temporal gyrus, inferior frontal gyrus, temporal pole and rolandic operculum. Error bars indicate $1 \mathrm{~s}$.e.m. fMRI, functional magnetic resonance imaging; FWE, family-wise error; MDD, major depressive disorder; TFCE, threshold-free cluster enhancement.

activity increases in the no-relapse group, whereas activity in the relapse group decreased from baseline to follow-up - mainly represented in the insula, temporal pole, temporal gyri, striatal and thalamic areas, as well as in the prefrontal cortex, including the anterior cingulate cortex (ACC), inferior, middle and superior frontal gyrus. There was no significant main effect of time at the whole-brain level $\left(P_{\text {TFCE-FWE }}=0.148\right)$.

\section{Cross-sectional group differences at baseline}

Supplementary Table 5 presents the results of the between-group comparisons in baseline brain function at the whole-brain level. There was a significant main effect of group at baseline, resulting from significantly lower baseline activity in the no-relapse group compared with the relapse group (Supplementary Fig. 4) and healthy controls in regions involving prefrontal and temporal regions, basal ganglia, cerebellum and the precentral gyrus.
Cross-sectional group differences at follow-up

At follow-up, there was no significant main effect of group at the whole-brain level $\left(P_{\text {TFCE-FWE }}>0.999\right)$.

\section{Additional analyses}

Effects of treatments on changes in brain function

Detailed results can be found in Supplementary Results 2.1 and Supplementary Table 6. Briefly, after controlling for medical and psychotherapeutic treatments, there was still a significant relapsextime interaction for the amygdala and insula ROIs.

\section{Relapse prediction}

The results of additional analyses investigating the predictive value of baseline brain functional data compared with clinical data are described in Supplementary Results 2.2 and Supplementary Table 7. The model including both clinical and baseline brain 
functional data revealed the best prediction regarding subsequent relapses compared with clinical or brain functional data alone. Significant predictors were cumulative duration of depression before baseline and baseline left insula activity.

\section{Effects of remission status on follow-up brain activity}

The division of the relapse group into two subgroups according to the remission status at follow-up resulted in $n=14$ patients with relapse in full remission and $n=33$ patients with relapse in current depression. The detailed results regarding the effects of remission status on follow-up brain activity can be found in Supplementary Results 2.3. Briefly summarised, there was a significant main effect of subgroup for the insula ROI (Fig. 3), resulting from lower activity in the relapse group with current depression compared with the healthy control group, as well as the norelapse group and the relapse group in full remission.

\section{Discussion}

The present study examined brain function during negative emotion processing in the long-term course of depression in a naturalistic 2-year follow-up design. The main finding was that patients with diverging course of depression showed different changes in brain activity during negative emotion processing within the amygdala, insula and temporal and prefrontal cortex. Although the majority of patients were receiving treatment, medication dose and psychotherapy did not affect the pattern of results in the amygdala or insula. Baseline differences in amygdala, hippocampus and insula activity were detected between patient groups with different subsequent course of illness.

\section{Changes in amygdala and insula activity}

Both the amygdala and the insula are regions relevant for the detection and processing of emotionally salient stimuli. ${ }^{2,22}$ The association of the insula with the course of illness is also highlighted by a previous study ${ }^{14}$ that showed reduced cortical surface area of the insula to be associated with future depressive relapse. In our study, the insula appears to be most sensitive for brain functional changes during emotion processing in the long-term course of illness. Increased insula activity in the no-relapse group seems to represent a normalisation of brain function, as activity was abnormally low at baseline during the acute episode and showed no difference at follow-up compared with healthy controls. Furthermore, healthy controls also showed a descriptive tendency for activity increases. Likewise, we found increased amygdala activity in the no-relapse group in response to negative stimuli after 2 years. Accordingly, Ai et $\mathrm{al}^{9}$ reported increased amygdala activity in association with symptom improvement during the encoding of positive and negative words. Combining these results, long-term remission may be associated with an increase in amygdala activity in response to emotional stimuli, independent of emotional valence. For the insula on the other hand, the changes in activity appear to be valence-specific, as we found increased activity in response to negative stimuli in patients in remission, whereas Opmeer et $\mathrm{al}^{10}$ reported reduced activity in response to positive stimuli in remitters. It should be noted, however, that there are differences in types of task and control conditions across studies, limiting the comparability of the results and interpretations regarding emotional valence.

\section{Changes in frontotemporal activity}

Our whole-brain results further reveal changes within the temporal pole, orbitofrontal and middle frontal gyrus, and within the dorsal part of the ACC. The temporal pole is involved in face recognition and plays a key role in the integration of visual input with semantic information. ${ }^{23}$ It has been reported to produce functional responses comparable to the amygdala, and is also activated during emotionally salient stimuli. ${ }^{23}$ In contrast to the amygdala and insula, the temporal pole is also involved in more complex socioemotional functions: ${ }^{23}$ temporal pole activity is evoked during theory-of-mind tasks and atrophies are associated with social withdrawal, abnormal social behaviour and lack of empathy. ${ }^{24}$ Our results indicate that patients in full remission show increased temporal pole activity during negative emotional face processing, which may depict enhanced integration of emotional and social information. In contrast, patients with relapse or ongoing depression show decreased activity of the temporal pole, suggesting further difficulties in activating socioemotional functions. One potential mechanism might be depression-associated social withdrawal and social anhedonia leading to a decrease in social interactions and, therefore, to deficits in employing mentalising and theory-of-mind capacities. However, as theory-of-mind tasks were not used in our study, this interpretation remains to be verified by future studies using more complex socioemotional paradigms.

Orbitofrontal, medial prefrontal areas, as well as the insula, are structurally connected with the temporal pole by the uncinate fasciculus, explaining the similar activation patterns. Remissionassociated activity increases in the orbitofrontal, prefrontal and dorsal cingulate cortex further suggest enhanced emotion regulation in the no-relapse group at follow-up, as stated by the emotion regulation model by Phillips et al. ${ }^{25}$ This finding is supported by a previous study that showed an association between symptom improvement after 6 months and an increase in prefrontal activity during regulation of negative affect. ${ }^{26}$ Further, a meta-analysis found the dorsal ACC and anterior insula to be the only regions involved in both explicit and implicit emotion regulation strategies. ${ }^{27}$ The ACC, inferior frontal gyrus extending to the insula, as well as the thalamus, are furthermore activated in applying the optimal policy in approach-avoidance conflicts, whereas activity of the amygdala and DLPFC is associated with a simpler heuristic strategy that considers only the probability of threat. ${ }^{28}$ Thus, the regions in which we found changes over time as a function of the course of depression also play an important role in the resolution of approach-avoidance conflicts in addition to emotion regulation.

Since structural neuroimaging studies found changes that are dependent on the course of illness in grey matter volume and cortical thickness in the insula, ACC, DLPFC and orbitofrontal cortex, ${ }^{13}$ the findings suggest that in the long term, depressive relapses are associated with reductions not only in grey matter volume, but also in brain activity during negative emotion processing.

\section{Effects of medication dose and psychotherapy on changes in brain activity}

Corresponding with previous long-term fMRI research, ${ }^{9}$ treatment with psychotherapy during the study interval did not influence the pattern of results. Medication load diminished the relapse-associated changes in brain function at the whole-brain level. Therefore, activity changes could partly be influenced by medication dose effects, which would be in line with short-term treatment studies revealing brain functional changes during negative emotion processing after antidepressant pharmacotherapy. ${ }^{6,8}$ Nevertheless, within the amygdala and insula, neither psychotherapy nor medication influenced the pattern of results. This suggests that the course of illness rather than specific effects of treatments may underlie long-term changes in amygdala and insula activity. 


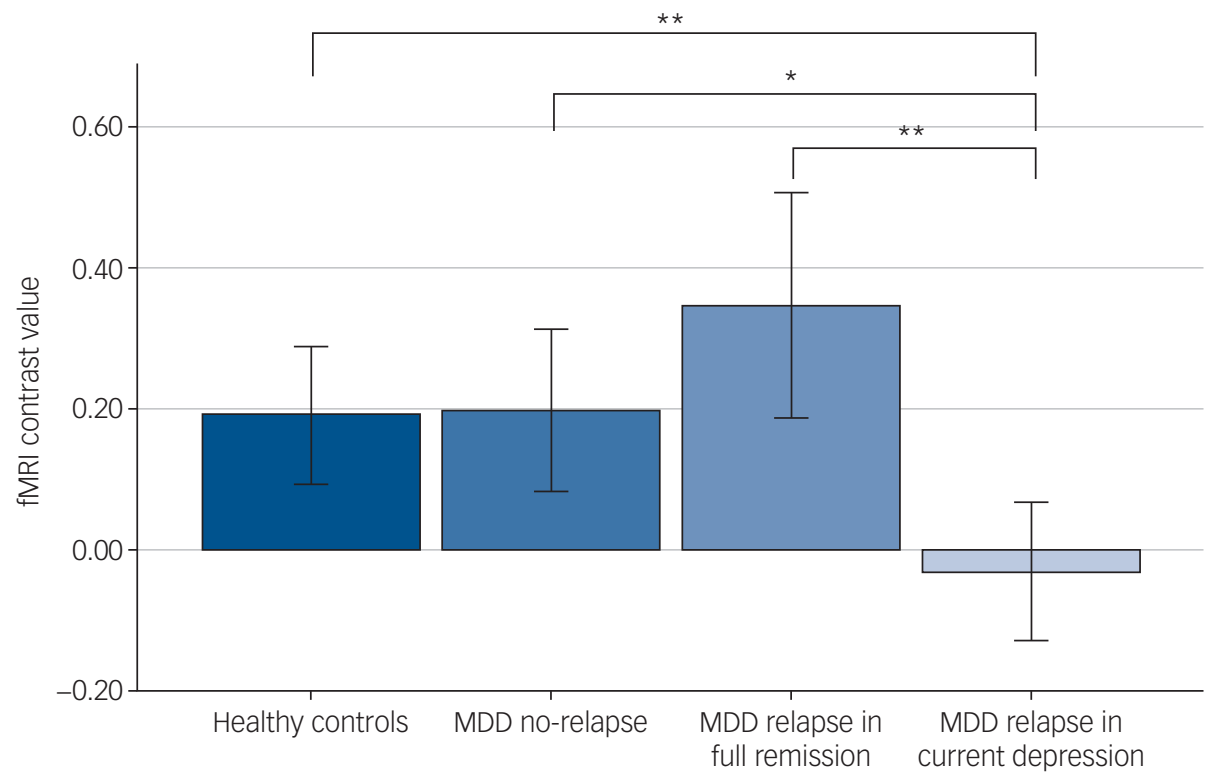

Fig. 3 Bar graph depicting typical insula responses (faces > shapes) at follow-up for subgroups divided by remission status. Error bars represent $95 \%$ confidence intervals. fMRI contrast values were computed by extracting the first Eigenvariate of the significant cluster resulting from the insula ROI analysis investigating the main effect of subgroup $\left(x=40, y=26, z=-6, F_{(3,110)}=7.00, k=11, P_{\mathrm{TFCE}-\mathrm{FWE}}=0.043\right)$ at follow-up of the oneway ANOVA. fMRI, functional magnetic resonance imaging; FWE, family-wise error; MDD, major depressive disorder; ROI, region of interest; TFCE, threshold-free cluster enhancement. ${ }^{*} P_{\mathrm{TFCE}-\mathrm{FWE}}<0.05, * * P_{\mathrm{TFCE}-F \mathrm{WE}}<0.01$.

\section{Cross-sectional differences in brain activity}

In contrast to previous studies, ${ }^{2,3}$ there was no difference in baseline amygdala, hippocampus and insula activity between the complete patient group including all patients with depression (both norelapse and relapse) and healthy controls. Dividing the patient group into subgroups according to the course of illness revealed that patients without future relapse showed abnormally low amygdala, hippocampus and insula activity compared with healthy controls and the relapse group. This heterogeneity in baseline brain function within patients with depression may provide a rationale for the missing difference between the complete patient group and healthy controls at baseline.

Our analyses revealed that the inclusion of baseline brain functional data substantially improved the prediction of relapse based on clinical data alone. These results underline the potential of fMRI as an additional predictive marker to identify patients at high risk of relapse.

The finding of higher baseline brain activity in patients with an unfavourable subsequent course of illness is supported by shortterm treatment studies that also suggest higher baseline activity of the insula ${ }^{29}$ and amygdala ${ }^{30}$ is associated with subsequent nonresponse to pharmacotherapy. In contrast, high psychological resilience has been found to be linked with lower amygdala activity during rest, ${ }^{31}$ and insula activity has been shown to differentiate between high-resilient and low-resilient individuals. ${ }^{32}$ The lower limbic activity detected in the no-relapse group could therefore be a function of higher resilience to subsequent depressive relapses. However, as the majority of patients included in our study was already under psychopharmacological treatment at baseline, lower baseline activity in the no-relapse group could also be a result of antidepressants applied before baseline. For example, studies showed that with antidepressant treatment, reductions in amygdala responses to negative stimuli occurred early, and even before the onset of mood changes, ${ }^{33}$ and that greater early reductions in amygdala and insula activity after antidepressant treatment were predictive for later response. ${ }^{34}$
Notably, there was no difference in baseline amygdala, hippocampus and insula activity between the relapse group and healthy controls. Again, psychopharmacological effects could have masked potential differences in brain function, reducing potential limbic hyperactivity in this patient group. Thus, antidepressants could potentially have diminished limbic hyperactivity in both groups of patients with depression (no-relapse and relapse), with only the no-relapse group showing neural correlates of early response in terms of limbic hypo-activity.

Taken together, these results suggest heterogeneity in the neural correlates of depression that may be associated with later disease progression, and therefore emphasise the importance of longitudinal phenotyping.

Exploratory analyses revealed that at follow-up, only patients with current depression had abnormal insula activity compared with healthy controls and patients in full remission. Instead, patients in full remission showed no difference in limbic activity compared with healthy controls, independent of the occurrence of relapses during the study interval. This finding demonstrates the importance of taking the mood state into account when investigating the course of illness, and is in line with cross-sectional studies showing mood-congruent brain functional alterations of the limbic system only in patients with current depression. ${ }^{2,35}$ Nevertheless, the small sample size per subgroup in our exploratory investigations should be considered and, subsequently, these results should be interpreted carefully.

\section{Strengths and limitations}

The major strengths of our study are the prospective naturalistic design providing a high external validity, the long study interval covering 2 years and a careful investigation of the course of illness. Nevertheless, some limitations should be mentioned. First, although we were able to examine dose-dependent effects of pharmacotherapy, the small number of drug-free patients did not allow us to examine the effect of medication versus no medication. Second, given the nature of our paradigm, it cannot be clearly stated 
whether the findings are a result of processing of the emotional valence of the faces or face processing in general. However, the paradigm applied in this study is well-established and frequently used to investigate brain functional correlates of emotion processing. ${ }^{16-18}$ Third, the reliability of paradigm-based fMRI is limited. ${ }^{36}$ Consequently, there is a high need for further longitudinal studies covering more participants, to improve the reliability of the results.

\section{Implications}

In summary, our study reveals long-term activity changes in emotion processing areas that are dependent on the course of illness, and point to a normalisation of brain function in remission. Our findings contribute to a more profound understanding of the brain functional correlates of conscious negative emotion processing in the context of depressive relapses, and thereby shed some light on the long-term neural trajectories of major depression. Common treatment approaches may be effective in reducing the negative bias in emotion processing.

Verena Enneking (D, MSC, Institute for Translational Psychiatry, University of Münster Germany; Melissa Klug, MSc, Institute for Translational Psychiatry, University of Münster, Germany; Tiana Borgers, MSc, Institute for Translational Psychiatry, University of Münster, Germany; Katharina Dohm, PhD, Institute for Translational Psychiatry, University of Münster, Germany; Dominik Grotegerd, PhD, Institute for Transtional Unverity of Muctional

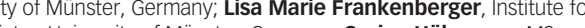
Translational Psychiatry, University of Münster, Germany; Carina Hülsmann, MSC, Institute for Translational Psychiatry, University of Münster, Germany; Hannah Lemke (D), MSc, Institute for Translational Psychiatry, University of Münster, Germany; Susanne Meinert, MSc, Institute for Translational Psychiatry, University of Münster, Germany; and Institute for Translational Neuroscience, University of Münster German, Elisabeth J. Leen,, DD, Instute for Transtion, Münster, Germany, Nils Opel, MD, Institute for Translational Psychiatry, University of Münster, Germany; Janik Goltermann, MSc, Institute for Translational Psychiatry, University of Münster, Germany; Maike Richter, MSc, Institute for Translational Psychiatry, University of Münster, Germany; Lena Waltemate, MSc, Institute for Translational Psychiatry, University of Münster, Germany; Joscha Böhnlein, PhD, Institute for Translational Psychiatry, University of Münster, Germany; Lisa Sindermann MSc, Institute for Translational Psychiatry, University of Münster, Germany; Jonathan Repple, MD, Institute for Translational Psychiatry, University of Münster, Germany; Jochen Bauer, PhD, Department of Clinical Radiology, University of Münster Germany; Mareike Thomas, MSc, Institute of Psychology, Martin-Luther University of Halle, Germany; Udo Dannlowski, MD, PhD, Institute for Translational Psychiatry, University of Münster, Germany; Ronny Redlich, PhD, Institute for Translational Psychiatry, University of Münster, Germany; and Institute of Psychology, Martin-Luther University of Halle, Germany

Correspondence: Ronny Redlich. Email: r.redlich@uni-muenster.de

First received 2 Jul 2021, final revision 17 Dec 2021, accepted 21 Dec 2021

\section{Supplementary material}

To view supplementary material for this article, please visit https://doi.org/10.1192/bjp.2021 223.

\section{Data availability}

The data that support the findings of this study are available from the corresponding author, R.R., upon reasonable request.

\section{Author contributions}

V.E., M.K., K.D., R.R. and U.D. contributed to the conception and design of the study. All authors contributed to the organisation of the conduct of the study (including acquisition of data). V.E., M.K., L.M.F., R.R. and U.D. analysed and interpreted the data. V.E., M.K., R.R. and U.D. drafted the work and all other authors revised it critically for important intellectual content. All authors have read and approved the final version of the manuscript.

\section{Funding}

The study was supported by grants from the Innovative Medizinische Forschung (grant RE111722 to R.R., grant I-KO121806 to K.D.), the German Research Foundation (grant RE4458/1-1 to R.R., grants FOR2107 DA1151/5-1 and DA1151/5-2 to U.D.) and the
Interdisciplinary Center for Clinical Research of the Medical Faculty of Münster (grant Dan3/ 012/17 to U.D.)

\section{Declaration of interest}

None.

\section{References}

1 Kessler RC, Berglund P, Demler O, Jin R, Koretz D, Merikangas KR, et al. The epidemiology of major depressive disorder: results from the National Comorbidity Survey Replication (NCS-R). JAMA 2003; 289(23): 3095-105.

2 Stuhrmann A, Suslow T, Dannlowski U. Facial emotion processing in major depression: a systematic review of neuroimaging findings. Biol Mood Anxiety Disord 2011; 1: 10

3 Groenewold NA, Opmeer EM, de Jonge P, Aleman A, Costafreda SG. Emotional valence modulates brain functional abnormalities in depression: evidence from a meta-analysis of fMRI studies. Neurosci Biobehav Rev 2013; 37(2): 152 63.

4 Kanai T, Takeuchi H, Furukawa TA, Yoshimura R, Imaizumi T, Kitamura T, et al. Time to recurrence after recovery from major depressive episodes and its predictors. Psychol Med 2003; 33(5): 839-45.

5 Ormel J, Oldehinkel AJ, Nolen WA, Vollebergh W. Psychosocial disability before, during, and after a major depressive episode: a 3-wave population-based study of state, scar, and trait effects. Arch Gen Psychiatry 2004; 61(4): 387-92.

6 Delaveau P, Jabourian M, Lemogne C, Guionnet S, Bergouignan L, Fossati P. Brain effects of antidepressants in major depression: a meta-analysis of emotional processing studies. J Affect Disord 2011; 130(1-2): 66-74.

7 Redlich R, Bürger C, Dohm K, Grotegerd D, Opel N, Zaremba D, et al. Effects of electroconvulsive therapy on amygdala function in major depression - a longitudinal functional magnetic resonance imaging study. Psychol Med 2017; 47 (12): 2166-76.

8 Ma Y. Neuropsychological mechanism underlying antidepressant effect: a systematic meta-analysis. Mol Psychiatry 2015; 20(3): 311-9.

9 Ai H, Opmeer EM, Marsman J-BC, Veltman DJ, van der Wee NJA, Aleman A, et al. Longitudinal brain changes in MDD during emotional encoding: effects of presence and persistence of symptomatology. Psychol Med 2020; 50(8): 131626.

10 Opmeer EM, Kortekaas R, van Tol M-J, Renken RJ, Demenescu LR, Woudstra S, et al. Changes in regional brain activation related to depressive state: a 2-year Iongitudinal functional MRI study. Depress Anxiety 2016; 33(1): 35-44.

11 Wittchen H-U, Wunderlich U, Gruschwitz S, Zaudig M. Strukturiertes Klinisches Interview für DSM-IV. Hogrefe, 1997.

12 Hamilton M. A rating scale for depression. J Neurol Neurosurg Psychiatry 1960; 23(1): 56-62.

13 Zaremba D, Dohm K, Redlich R, Grotegerd D, Strojny R, Meinert S, et al. Association of brain cortical changes with relapse in patients with major depressive disorder. JAMA Psychiatry 2018; 75(5): 484-92.

14 Opel N, Redlich R, Dohm K, Zaremba D, Goltermann J, Repple J, et al. Mediation of the influence of childhood maltreatment on depression relapse by cortical structure: a 2-year longitudinal observational study. Lancet Psychiatry 2019; 6 (4): $318-26$.

15 American Psychiatric Association. Diagnostic and Statistical Manual of Mental Disorders: DSM-IV-TR. American Psychiatric Association, 2000.

16 Redlich R, Grotegerd D, Opel N, Kaufmann C, Zwitserlood P, Kugel H, et al. Are you gonna leave me? Separation anxiety is associated with increased amygdala responsiveness and volume. Soc Cogn Affect Neurosci 2015; 10(2): 27884.

17 Dannlowski U, Stuhrmann A, Beutelmann V, Zwanzger P, Lenzen T, Grotegerd D, et al. Limbic scars: long-term consequences of childhood maltreatment revealed by functional and structural magnetic resonance imaging. Biol Psychiatry 2012; 71(4): 286-93.

18 Hariri AR, Mattay VS, Tessitore A, Kolachana B, Fera F, Goldman D, et al. Serotonin transporter genetic variation and the response of the human amygdala. Science 2002; 297(5580): 400-3.

19 Ekman P, Friesen WV. Pictures of Facial Affect. Consulting Psychologists Press, 1976

20 Maldjian JA, Laurienti PJ, Kraft RA, Burdette JH. An automated method for neuroanatomic and cytoarchitectonic atlas-based interrogation of fMRI data sets. Neuroimage 2003; 19(3): 1233-9.

21 Tzourio-Mazoyer N, Landeau B, Papathanassiou D, Crivello F, Etard O, Delcroix N, et al. Automated anatomical labeling of activations in SPM using a macroscopic 
anatomical parcellation of the MNI MRI single-subject brain. Neuroimage 2002 15(1): 273-89.

22 Phan KL, Wager T, Taylor SF, Liberzon I. Functional neuroanatomy of emotion: a meta-analysis of emotion activation studies in PET and fMRI. Neuroimage 2002; 16(2): 331-48

23 Olson IR, Plotzker A, Ezzyat Y. The enigmatic temporal pole: a review of find ings on social and emotional processing. Brain 2007; 130(7): 1718-31.

24 Gorno-Tempini ML, Rankin KP, Woolley JD, Rosen HJ, Phengrasamy L, Miller BL. Cognitive and behavioral profile in a case of right anterior temporal lobe neurodegeneration. Cortex 2004; 40(4-5): 631-44.

25 Phillips ML, Drevets WC, Rauch SL, Lane R. Neurobiology of emotion perception I: the neural basis of normal emotion perception. Biol Psychiatry 2003; 54(5): 504-14.

26 Heller AS, Johnstone T, Peterson MJ, Kolden GG, Kalin NH, Davidson RJ. Increased prefrontal cortex activity during negative emotion regulation as a predictor of depression symptom severity trajectory over 6 months. JAMA Psychiatry 2013; 70(11): 1181-9.

27 Picó-Pérez M, Alemany-Navarro M, Dunsmoor JE, Radua J, Albajes-Eizagirre A Vervliet $\mathrm{B}$, et al. Common and distinct neural correlates of fear extinction and cognitive reappraisal: a meta-analysis of fMRI studies. Neurosci Biobehav Rev 2019; 104: 102-15.

28 Korn CW, Bach DR. Minimizing threat via heuristic and optimal policies recruits hippocampus and medial prefrontal cortex. Nat Hum Behav 2019; 3(7): 733-45.

$29 \mathrm{Fu}$ CHY, Steiner H, Costafreda SG. Predictive neural biomarkers of clinical response in depression: a meta-analysis of functional and structural neuroimaging studies of pharmacological and psychological therapies. Neurobiol Dis 2013; 52: 75-83.
30 Williams LM, Korgaonkar MS, Song YC, Paton R, Eagles S, Goldstein-Piekarski A et al. Amygdala reactivity to emotional faces in the prediction of general and medication-specific responses to antidepressant treatment in the randomized iSPOT-D trial. Neuropsychopharmacology 2015; 40(10): 2398-408.

31 Leaver AM, Yang H, Siddarth P, Vlasova RM, Krause B, St. Cyr N, et al. Resilience and amygdala function in older healthy and depressed adults. J Affect Disord 2018: 237: 27-34.

32 Waugh CE, Wager TD, Fredrickson BL, Noll DC, Taylor SF. The neural correlates of trait resilience when anticipating and recovering from threat. Soc $\operatorname{Cog} n$ Affect Neurosci 2008; 3(4): 322-32.

33 Godlewska BR, Norbury R, Selvaraj S, Cowen PJ, Harmer CJ. Short-term SSR treatment normalises amygdala hyperactivity in depressed patients. Psychol Med 2012; 42(12): 2609-17.

34 Godlewska BR, Browning M, Norbury R, Cowen PJ, Harmer CJ. Early changes in emotional processing as a marker of clinical response to SSRI treatment in depression. Transl Psychiatry 2016; 6(11): e957.

35 Arnone D, McKie S, Elliott R, Thomas E, Downey D, Juhasz G, et al. Increased amygdala responses to sad but not fearful faces in major depression: relation to mood state and pharmacological treatment. Am JPsychiatry 2012; 169(8): 841-50.

36 Elliott ML, Knodt AR, Ireland D, Morris ML, Poulton R, Ramrakha S, et al. What is the test-retest reliability of common task-functional MRI measures? New empirical evidence and a meta-analysis. Psychol Sci 2020; 31(7): 792-806.

37 Eickhoff SB, Stephan KE, Mohlberg H, Grefkes C, Fink GR, Amunts K, et al. A new SPM toolbox for combining probabilistic cytoarchitectonic maps and functional imaging data. Neurolmage 2005; 25(4): 1325-35. 